\title{
Case Report of a Rare Incidence of IgH Amplification Leading to Acute Kidney Injury in a Multiple Myeloma Patient
}

\author{
Sowmya Thanikachalam ${ }^{a}$ Vijay Kumar Srinivasalu ${ }^{a, b} \quad$ K.S. Nataraj ${ }^{c}$ \\ Sharat Damodar ${ }^{\mathrm{C}}$ Manjula Das ${ }^{\mathrm{a}}$ \\ ${ }^{a}$ Tumor Immunology Program, Mazumdar Shaw Medical Foundation, Bangalore, India; \\ ${ }^{b}$ Department of Medical Oncology, Mazumdar Shaw Medical Centre, Bangalore, India; \\ 'Department of Hematology, Mazumdar Shaw Medical Centre, Bangalore, India
}

\section{Keywords}

Multiple myeloma · IgH amplification · Acute kidney injury · Molecular cytogenetics · Heavy chain deposition

\begin{abstract}
We present a case report of a 62-year-old male, treated for kappa light chain multiple myeloma with chemotherapy followed by autologous stem cell transplant (ASCT) in 2014. He has been in complete remission for 4 years. In 2018, he was evaluated for hypercreatinemia and acute kidney injury(AKI) with a suspicion of disease relapse; he underwent evaluation with bone marrow aspiration cytology which showed no evidence of relapse. However, careful cytogenetic analyses showed IgH amplification (14q32) which probably was the cause for AKI in the absence of any structural abnormality in the kidney. Heavy chain deposition leads to AKI in multiple myeloma, and its association with IgH amplification leading to AKI is reported here. Though heavy chain deposition leading to AKI is common, $\mathrm{IgH}$ amplification at chromosome level is the first case observed.

\section{Introduction}

Multiple myeloma is a cancer of plasma cells accounting for $10 \%$ of all hematological malignancies. Their clinical manifestations include anemia, bone lesions, renal dysfunction, and altered immune function. It is characterized by several complex chromosomal abnormalities. Frequently reported high-risk abnormalities include IgH translocations, deletion 
Table 1. Common chromosomal abnormality in multiple myeloma involving IgH

\begin{tabular}{llll}
\hline SN & Chromosomal abnormality & Clinical impact & Ref \\
\hline 1 & IgH translocations & Oncogene amplification of BCL2, BCL6, MYC & [1] \\
2 & 14q32 loss, $t(4 ; 14)$ deletion, $t(4 ; 14)$ amplification, 14 trisomy & & [2] \\
3 & IgH translocation with trisomy of odd chromosomes, monosomy 14 & High-risk abnormalities & [3] \\
4 & IgH translocations, del 1p32, 1q gain, hyperdiploidy and trisomy & High-risk abnormality & \\
\hline
\end{tabular}

Table 2. Amplification/trisomy 14 reported in other hematological malignancies

\begin{tabular}{llll}
\hline SN & Translocation details & Clinical implication & Ref \\
\hline 1 & IgH amplification with MYC amplification, IgH-BCL2 translocation & DLBCL unclassified, high risk & [4] \\
2 & Low-level IgH amplification & Higher survival with multiple relapses & [4] \\
3 & 14q24-32 amplification with $\mathrm{t}(11 ; 14)$, Tp53 del & Poor prognosis in MCL & [5] \\
\hline
\end{tabular}

1p32, 1q gains, hyperdiploidy, and trisomies of various gene loci. IgH translocations lead to several oncogene amplifications such as BCL2, BCL6, MYC, etc. [1]. IgH deletion/loss of IgH locus (14q32), amplification of IgH translocations such as IgH-FGFR3 fusion and trisomy of 14 have been reported [2]. IgH translocations with trisomy of odd chromosomes and monosomy of 14 are categorised as high-risk abnormalities, suggesting the importance of cytogenetics in multiple myeloma [3]. Common IgH translocations reported in multiple myeloma are summarized in Table 1.

IgH amplification, though not described in multiple myeloma, has been reported in other cancers, as presented in Table 2. IgH amplification with or without MYC amplification, IgH-CMYC translocation, and IgH-BCL2 translocations are reported in DLBCL-unclassified diseases. Among them, low-level IgH amplification had a higher survival with multiple relapses [4].14q 24-32 amplification accompanied with complex chromosomal/genetic alterations such as $\mathrm{t}(11 ; 14)$, monoallelic deletion of Tp53, and TP53 mutations are reported in mantle cell lymphoma leading to poor prognosis [5].

Here, we present molecular cytogenetics of a renal injury patient who was a known case of multiple myeloma revealing mosaic of $14 \mathrm{q} 32$ (high) amplification in $\approx 30 \%$ of cells.

\section{Case Report}

A 62-year-old gentleman evaluated for acute onset of low back pain in January 2014 was found to have lumbar spine fracture, later diagnosed to have due to kappa light chain multiple myeloma, ISS stage III with high-risk cytogenetics. He was treated with radiotherapy to the lumbar spine followed by 4 cycles of chemotherapy with VRd regimen, bortezomib (D1, D8, D15, and D22 each cycle), lenlidomide, and dexamethasone followed by ASCT in June 2014. He was in complete remission for 4 years with his free light chain ratio in normal limits. In December 2018, he presented to the emergency department with reduced urine output and elevated serum creatinine $(1.8 \mathrm{mg} / \mathrm{dL})$. He was evaluated with urine routine and culture tests, ultrasound (USG) of abdomen and pelvis, serum protein electrophoresis (SPEP), and a bone marrow (BM) aspiration and biopsy to rule out a myeloma relapse. Urine routine was normal with no evidence of pus cells. Urine culture was sterile with no growth after $48 \mathrm{~h}$ of incubation. Complete blood count report showed hemoglobin $13.0 \mathrm{~g} / \mathrm{dL}$, platelet count $156,000 / \mu \mathrm{L}$, and total leukocytes 6,300 cells $/ \mathrm{mm}^{3}$ with $70 \%$ neutrophils. There was no 
Fig. 1. Interphase nuclear FISH using IgH BA probe demonstrates amplification of IgH locus (3 copies).

Fig. 2. Interphase nuclear FISH using $\mathrm{t}(14 ; 16)$ probe demonstrates amplification of IgH locus (3 copies).

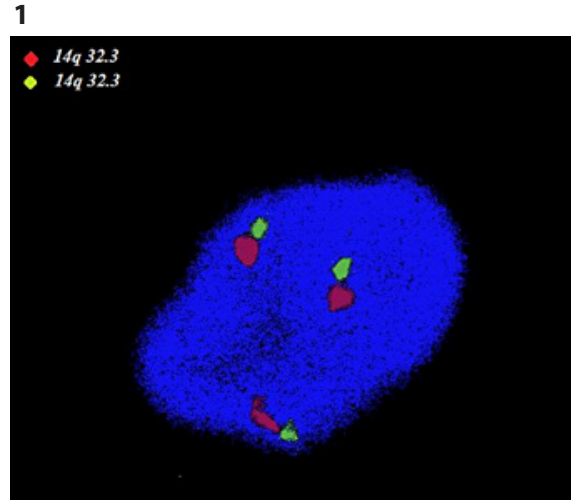

2

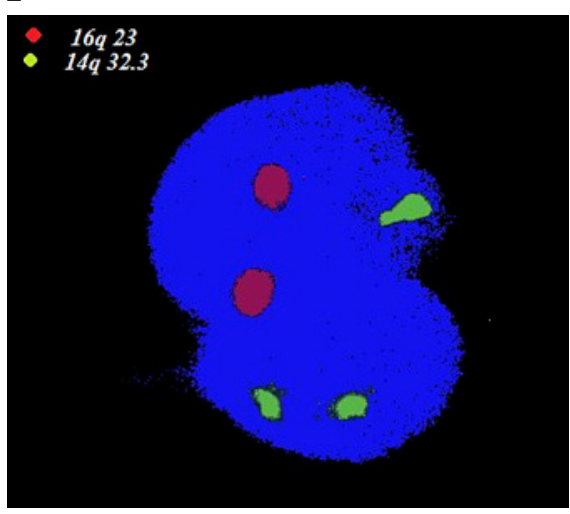

3

Fig. 3. Interphase nuclear FISH using $\mathrm{t}(4 ; 14)$ probe demonstrates amplification of IgH locus (3 copies).

Fig. 4. Interphase nuclear FISH using $\mathrm{t}(14 ; 20)$ probe demonstrates amplification of IgH locus (3 copies).

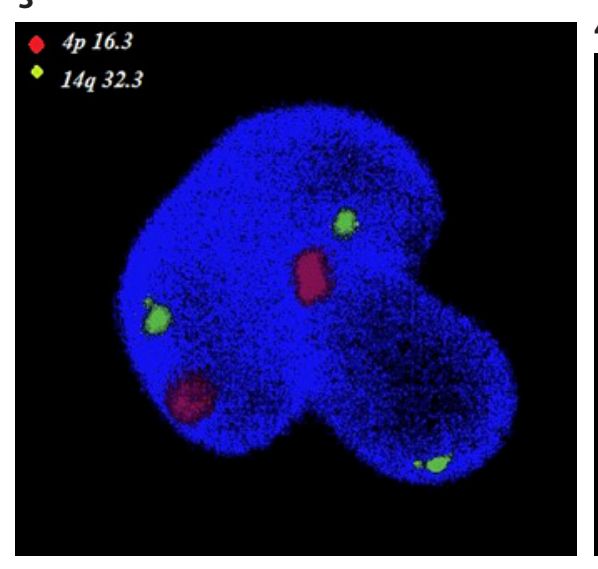

evidence of neutrophilic leukocytosis. Corrected serum calcium was $9.3 \mathrm{mg} / \mathrm{dL}$. USG of abdomen showed no evidence of bladder wall thickening or cystitis. Both kidneys were normal in size with maintained corticomedullary differentiation. SPEP showed an M-spike of $0.62 \mathrm{mg} / \mathrm{dL}$. BM report showed no definitive evidence of myeloma relapse. He was treated for acute kidney injury (AKI) with IV hydration. BM aspirate for molecular cytogenetic testing found IgH amplification (14q32) in 30\% of cells. In previously reported multiple myeloma cases, IgH translocation, monosomy and hyperdiploidy of odd chromosomes have been reported. IgH amplification has not been mentioned in genetics-based risk stratification in myeloma [6, 7]. The patient did not show any evidence of infection with acute kidney damage and medium expression of LCN2. In view of absence of possible explanation for elevated serum creatinine, we believe IgH amplification is the most probable explanation for acute renal failure, as it would lead to heavy chain deposition in kidney leading to kidney damage.

\section{Molecular Cytogenetic Panel}

BM sample was collected in a sodium heparin vacutainer (BD Biosciences, India) and processed within $6 \mathrm{~h}$ of aspiration. To $1.5 \mathrm{~mL}$ of BM, hypotonic treatment was given with prewarmed 0.075 M KCL (Sigma Aldrich, USA), followed by fixation with prechilled Carnoy's fixative (3:1 methanol, Himedia, India, and acetic acid, Fisher Scientific). Fixed cells were then processed for FISH analysis using Tp53 deletion (D-5103-100-OG), 14q32.3 (D-5107100-OG), t(4; 14) (D-5108-100-OG), t(14; 20) (D-5105-100-OG), t(14; 16) (D-5112-100-OG), 13q14.2(RB1) deletion (D-5070-100-TC), 1p32.3 amplification/1q21.22 deletion (D-5099- 
100-OG), and 5p15/15q22 hyperdiploidy probes (D-5095-100-TC) from Metasystems, Germany. The protocol was done as described by the company. Briefly, cells were fixed onto a slide, denatured at $72 \pm 2^{\circ} \mathrm{C}$ for $2 \mathrm{~min}$, hybridized overnight at $37^{\circ} \mathrm{C}$ in a humidified chamber followed by posthybridization washes with $0.4 \times \mathrm{SSC}$ (tri-sodium citrate and sodium chloride, Himedia) at $72^{\circ} \mathrm{C}$ for $2 \mathrm{~min}, 2 \times \mathrm{SSC}$ with $0.05 \%$ tween-20 (Himedia) for $1 \mathrm{~min}$ followed by a rinse in deionized distilled water. Slides were then air dried and counterstained with DAPI (Metasystems, Germany) and analyzed using Carl Zeiss Axio scope A1 and Zen Imaging software (Carl Zeiss).

Among the FISH panel, IgH BA probe revealed IgH amplification ( 3 copies) in $30 \%$ of the cells. The IgH BA results were complemented by $t(4 ; 14), t(14 ; 16)$, and $t(14 ; 20)$ probes and were negative forFGFR3/MAF/MAFB amplification or deletion (Figs. 1-4). FISH for chromosome 17/17p13 deletion, 13q14.2(RB1) deletion, 1p32.3 amplification/1q21.22 deletion, and $5 \mathrm{p} 15 / 15 \mathrm{q} 22$ hyperdiploidy was negative (data not shown).

LCN2 level in the serum was checked using ELISA developed in-house. LCN2 level is a known kidney injury/infection marker, generally high in the case of infection and medium in case of injury. The patient showed medium expression of LCN2 indicating kidney injury and not infection.

The patient is on regular follow-up, and his serum free light chain assay and SPEP have been found to be normal as of March 2019. He was started on lenalidomide and dexamethasone as maintenance therapy, and his creatinine level was under control as of May 2019.

\section{Discussion}

The probable reason for the AKI would be due to heavy chain deposition in the organ. Unlike the light chain disease, the heavy chain disease could not be detected with protein electrophoresis and shows hypogammoglobulinemia. Confirmation of heavy chain deposition has to be done by immunostaining and immunoelectrophoresis of IgA levels. Heavy chain-associated myelomas have been reported previously and lead to end-stage renal disease in a slow process $[8,9]$. The diagnosis is supported by the medium expression of LCN2 levels, which is a kidney injury marker as opposed to high expression representing kidney infection. Also, the urine culture reports reveal no growth, suggesting that the AKI is due to the IgH amplification and not due to the infection.

AKI in multiple myeloma is usually due to deposition of abnormal immunoglobulins in the kidney, but we report a case of IgH amplification as the cause of AKI in a patient with multiple myeloma in the absence of any structural abnormality in the kidney. We hypothesize that IgH amplification can cause genetic alteration, resulting in AKI.

\section{Acknowledgement}

The case report was funded in part by the host organisation. We thank Sreejeta Mondal for collection of sample and consent form.

\section{Statement of Ethics}

Informed consent has been obtained from the patient to use his sample for research and publication of the findings as per institutional ethics norms.

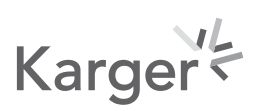




\section{Conflict of Interest Statement}

The authors have no conflict of interest.

\section{Funding Sources}

The case report is funded by Mazumdar Shaw Medical Foundation.

\section{Author Contributions}

S.T. and V.K.S.: conceptualization and manuscript writing; K.S.N. and S.D.: patient sample and clinical guidance; M.D.: - coordination and editing.

\section{References}

1 Sun L, Montella L. Oncogene Amplification as an Incidental Finding in FISH Testing for Gene Rearrangements in Lymphoid Hematopoietic Neoplasms. Blood. 2011;118(21):2505-5.

2 Smol T, Daudignon A. Comparison of IGH Profile Signals Using t(4;14) and IGH Break-Apart Probes by FISH in Multiple Myeloma. Cytogenet Genome Res. 2017;153:18-21.

3 Kumar S, Fonseca R, Ketterling RP, Dispenzieri A, Lacy MQ, Gertz MA, et al. Trisomies in multiple myeloma: impact on survival in patients with high-risk cytogenetics. Blood. 2012;119(9):2100-5.

4 Bellone M, Zaslav AL, Ahmed T, Lee HL, Ma Y, Hu Y. IGH amplification in patients with B cell lymphoma unclassifiable, with features intermediate between diffuse large B cell lymphoma and Burkitt's lymphoma. Biomark Res. 2014;2:9.

5 Gruszka-Westwood AM, Atkinson S, Summersgill BM, Shipley J, Elnenaei MO, Jain P, et al. Unusual case of leukemic mantle cell lymphoma with amplified CCND1/IGH fusion gene. Genes Chromosomes Cancer. 2002; 33(2):206-12.

6 Le GN, Bones J, Coyne M, Bazou D, Dowling P, O'Gorman P, et al. Current and future biomarkers for risk-stratification and treatment personalisation in multiple myeloma. Molecular Omics. 2019;15:7-20.

7 Ria R, Dammacco, F and Vacca A. Heavy-Chain Diseases and Myeloma-Associated Fanconi Syndrome: an Update. Mediterr J Hematol Infect Dis 2018;10(1):e2018011.

8 Ma CX, Lacy MQ, Rompala JF, Dispenzieri A, Rajkumar SV, Greipp PR, et al. Acquired Fanconi syndrome is an indolent disorder in the absence of overt multiple myeloma. Blood. 2004;104(1):40-2.

9 Rane S, Rana S, Mudrabettu C, Jha V, Joshi K. Heavy-chain deposition disease: a morphological, immunofluorescence and ultrastructural assessment. Clin Kidney J. 2012;5(5):383-9. 